\title{
DIFFÉRENTES MÉTHODES D’APPRENTISSAGE ACTIF POUR UNE DIVERSITÉ D’APPRENANTS
}

Yves Boudreault

Polytechnique Montréal

yves.boudreault@polymtl.ca

Lina Forest

Polytechnique Montréal

lina.forest@polymtl.ca

\author{
Catherine Carré \\ Polytechnique Montréal \\ catherine.carret@polymtl.ca
}

Pierre G. Lafleur

Polytechnique Montréal

pierre.lafleurt@polymtl.ca

\author{
Patrice Farand \\ Polytechnique Montréal \\ patrice.farand@polymtl.ca
}

\author{
Annie Ross \\ Polytechnique Montréal \\ annie.ross@polymtl.ca
}

Résumé - Pédagogie active, enseignement actif ou apprentissage actif, sont des termes qui ont comme point commun l'activité d'apprentissage proposée à l'étudiant, pour qu'il s'engage dans son processus d'apprentissage. La pédagogie active offre un défi intéressant à l'enseignant qui doit concevoir son enseignement comme une scénarisation de situations d'apprentissage. Depuis plusieurs années, les méthodes d'enseignement liées à la pédagogie active sont prisées à Polytechnique Montréal. Ainsi, à chaque année depuis 10 ans, des enseignants sont invités à présenter à leurs pairs leur expérience innovatrice d'enseignement dans le cadre d'une journée vouée à l'enseignement et à l'apprentissage. L'objectif de cet article est de présenter deux expériences de classe inversée et de souligner leur impact sur l'étudiant en ingénierie à l'aide d'observations.

Mots clés : méthodes d’apprentissage; apprentissage actif; situations d’apprentissage; classe inversée; MOOC.

\section{INTRODUCTION}

Nous concevons tous que l'enseignement ne se résume pas à une interaction entre l'enseignant, diffuseur de connaissances, et l'étudiant, récepteur de connaissances. Le passage d'un enseignement centré sur l'enseignant vers un enseignement centré sur l'apprenant modifie les rôles de l'enseignant et de l'apprenant. L'enseignant doit proposer des activités qui permettront à l'étudiant de s'investir dans un processus d'apprentissage fertile. L'étudiant devient ainsi responsable de son apprentissage en adoptant le rythme qui lui convient et en utilisant les techniques d'apprentissage qui lui sont appropriées.

Les enseignants désirant s'engager dans une pédagogie active s'interrogent, pour la plupart, par le choix de la méthode d'enseignement à privilégier. De plus, ils se demandent comment un apprentissage actif se distingue d'un enseignement traditionnel en ingénierie puisque l'étudiant est actif lors des travaux pratiques en laboratoire ou lors des séances de travaux dirigés.
En guise de réponse à ces interrogations, cet article présente deux expériences de classe inversée, en identifiant leurs caractéristiques et leur impact sur l'apprentissage de l'étudiant.

\section{DÉFINITIONS}

L'apprentissage actif s'inscrit dans une méthode d'enseignement qui engage l'étudiant dans son processus d'apprentissage. Pour ce faire, l'enseignant scénarise une ou des situations actives d'apprentissage. La situation d'apprentissage peut être réalisée par un groupe d'étudiants, ce qui est généralement le cas en classe, ou bien individuellement.

Les méthodes d'enseignement actif se distinguent selon différents critères, dont l'accompagnement de l'enseignant et l'interaction entre les étudiants. L’accompagnement se définit, entre autres, par l'intensité, la nature et le moment d'intervention de l'enseignant. L'intensité précise le pourcentage de temps de disponibilité de l'enseignant par rapport à la durée 
totale de la situation. La nature précise comment l'enseignant soutient les étudiants dans leur démarche. Finalement, le moment indique à quelle période dans la situation d'apprentissage l'enseignant se manifeste. Pour ce qui est de l'interaction entre les étudiants, nous nous limiterons à distinguer s'ils adoptent une stratégie coopérative en divisant le travail entre les coéquipiers ou une stratégie collaborative en réalisant tous ensemble chaque étape du travail. Dans les deux cas, les capacités de communication et d'interaction entre les étudiants sont fortement sollicitées.

\section{Deux cours en classe inversée}

\subsection{MEC2420 - Dynamique de l’ingénieur}

Le cours MEC2420 - Dynamique de l'ingénieur est un cours de deuxième année du programme de génie mécanique qui accueille environ 200 étudiants, au trimestre d'automne et au trimestre d'hiver, répartis en 3 groupes-cours et 5 groupes de travaux pratiques en laboratoire. Ce cours présente les concepts fondamentaux de la mécanique appliqués à l'analyse des mécanismes et des éléments de machine. L’horaire hebdomadaire de la classe traditionnelle est de cinq heures, trois heures sont consacrées à la théorie et deux heures sont consacrées à la pratique. À cela s’ajoutent quatre heures de travail personnel.

Dans le mode de la classe inversée, les heures de travail personnel sont consacrées à l'assimilation des notions théoriques à l'aide, entre autres, du visionnement de capsules vidéo. Les cinq heures de cours hebdomadaire sont réparties différemment. Les activités de la première heure sont : un retour sur la ou les capsules, la réponse à un questionnaire formatif en ligne, constitué de questions de compréhension, et le dévoilement des «bonnes» réponses aux questions. À la deuxième et troisième heure, les étudiants doivent réaliser seuls ou en équipe des exercices d'application (environ 3 à 5 exercices) sous la supervision de l'enseignante. Cette activité est suivie d'une mise en commun des solutions, tout en explicitant les détails d'exécution et en soulignant les pièges et les erreurs fréquentes à éviter. Finalement, les quatrième et cinquième heures sont consacrées à la résolution de problèmes (généralement 2 à 3 problèmes), également en équipe, et se terminent par un exercice individuel évalué d’une durée de 15 minutes. Il nous apparaît ici nécessaire de distinguer la réalisation d'un exercice de la réalisation d'un problème. La réalisation d'un exercice correspond à appliquer une technique, un algorithme en fonction des données soumises. La réalisation d'un problème nécessite, quant à elle, la recherche de la technique ou de l’algorithme qui permettra de le résoudre.

\subsubsection{Résultats et observations}

Les résultats de l'évaluation des apprentissages de l'automne 2015 (A2015) sont comparés à ceux de l'automne 2013 (A2013), parce qu'il s'agit de la même enseignante responsable du cours, et de l'automne 2009 (A2009) parce qu’il y avait également des exercices évalués à ce trimestre.

Les résultats globaux démontrent une meilleure réussite des étudiants du point de vue de la moyenne (sur 4) de l'ensemble des étudiants inscrits et du nombre d'étudiants ayant réussi le cours. Le tableau 1 montre que le taux de réussite des étudiants de l'A2015 est supérieur de 9,4 points de pourcentage par rapport à celui de l'A2013 et de 10,2 par rapport à celui de l'A2009.

Tableau 1. Moyenne et réussite au cours MEC2420 de chaque cohorte

\begin{tabular}{|l|l|l|l|}
\cline { 2 - 4 } \multicolumn{1}{c|}{} & A2015 & A2013 & A2009 \\
\hline $\begin{array}{l}\text { Moyenne } \\
\text { (sur 4) }\end{array}$ & 2,22 & 1,80 & 1,84 \\
\hline Réussite & $86,6 \%$ & $77,2 \%$ & $76,4 \%$ \\
\hline
\end{tabular}

À la figure 1, nous pouvons observer un nombre plus élevé d'étudiants dans l'intervalle $[A *, B]$ et un nombre plus faible d'étudiants dans l'intervalle $[\mathrm{D}+\mathrm{F}]$, où $\mathrm{F}$ correspond à un échec, pour la cohorte de l'A2015 par rapport aux deux autres cohortes. Il s'agit, sans contredit, du résultat souhaité par l'enseignante qui incite les étudiants à s'investir dans leur apprentissage par divers moyens de la classe inversée. Soulignons que dans la figure 1, la note $A B$ est attribuée aux étudiants ayant abandonné le cours.

Figure 1. Répartition des notes pour chaque cohorte

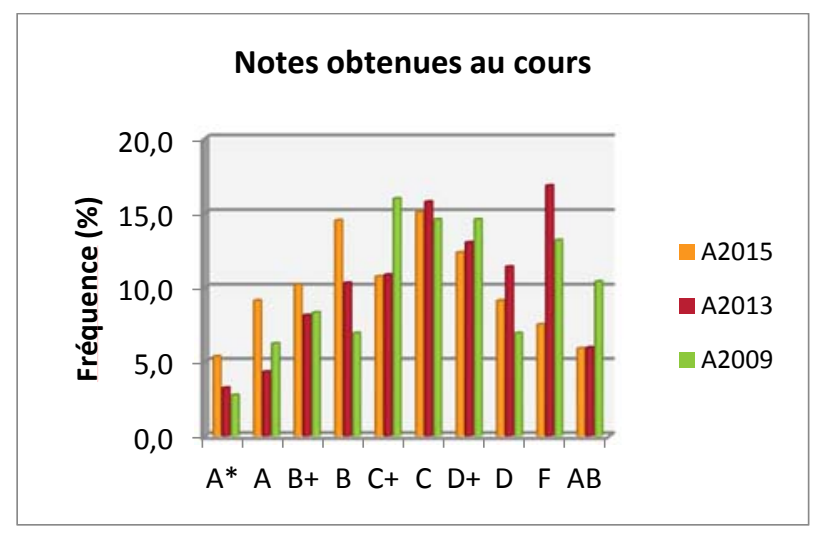


La figure 2 et le tableau 2 présentent respectivement les notes obtenues par les étudiants à l'examen final et celles des exercices évalués.

Dans le graphique de la figure 2, pour l'intervalle [61,70], on voit clairement la présence d'un plus grand nombre d'étudiants de la cohorte A2015 par rapport aux deux autres cohortes. Une augmentation du double et même du triple. À notre avis, il s'agit d'une donnée intéressante puisque cela correspond à un déplacement de la zone des étudiants qui ne réussissent pas le cours vers la zone des étudiants qui réussissent le cours.

Figure 2. Notes obtenues à l'examen final

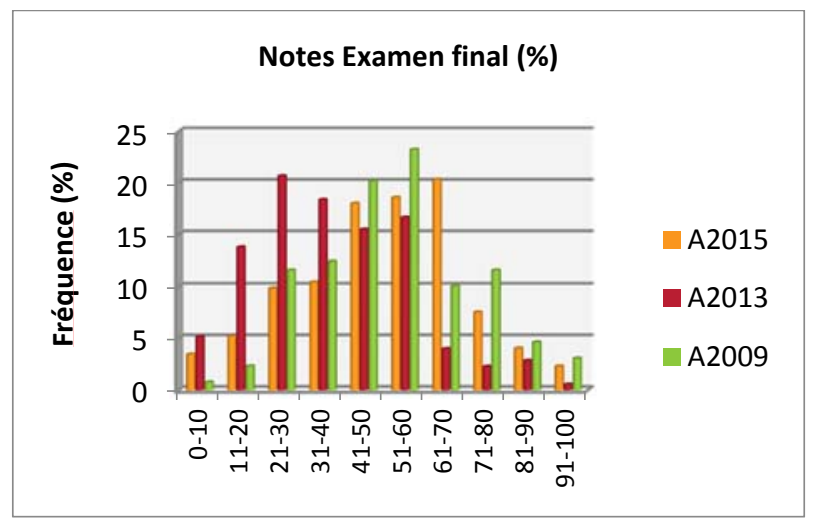

Les résultats aux exercices évalués, pour les deux trimestres où cette évaluation a été réalisée, sont comparés au tableau 2. On observe 14 points de pourcentage de plus pour les étudiants de l'A2015 par rapport à ceux de l'A2009 pour l'intervalle $[0,2]$ et 10 points de pourcentage de plus pour la note maximale de 5. Encore une fois, il s'agit d'un résultat à la faveur d'un enseignement où l'étudiant est profondément engagé dans son processus d'apprentissage. Il n'a pas le choix, il doit se présenter aux séances et réaliser les exercices. La dynamique de l'équipe et de l'ensemble du groupe peut favoriser l'appropriation des notions par l'étudiant.

Tableau 2. Notes obtenues aux exercices évalués

\begin{tabular}{|l|l|l|l|l|}
\hline \multirow{2}{*}{ Note } & \multicolumn{2}{|c|}{ A2009 } & \multicolumn{2}{c|}{ A2015 } \\
\cline { 2 - 5 } & $\mathrm{N}$ & $\%$ & $\mathrm{~N}$ & $\%$ \\
\hline $5 / 5$ & 32 & 23 & 57 & 33 \\
\hline $3 / 5-4 / 5$ & 45 & 32 & 62 & 36 \\
\hline $0 / 5-2 / 5$ & 63 & 45 & 54 & 31 \\
\hline Total & 140 & 100 & 173 & 100 \\
\hline
\end{tabular}

Dans l'ensemble, l'analyse préliminaire des résultats obtenus aux évaluations montre une meilleure réussite des étudiants de la cohorte 2015A, dont l'enseignement est réalisé en classe inversée. Cependant, les étudiants ont-ils apprécié l’approche de la classe inversée? Pour répondre à cette question, les étudiants ont répondu à un questionnaire, dont les résultats suivent.

Tout d'abord, la figure 3 montre l'utilité, selon les étudiants, des différents dispositifs mis à leur disposition: capsules vidéo, manuel, exercices notés et exercices en classe. Sans surprise, le manuel apparaît inutile puisqu'il est remplacé par les vidéos. À l'opposé, les exercices en classe sont grandement appréciés. Les capsules vidéo et les exercices notés sont également considérés comme utiles par les étudiants.

Figure 3 Utilité des dispositifs pédagogiques

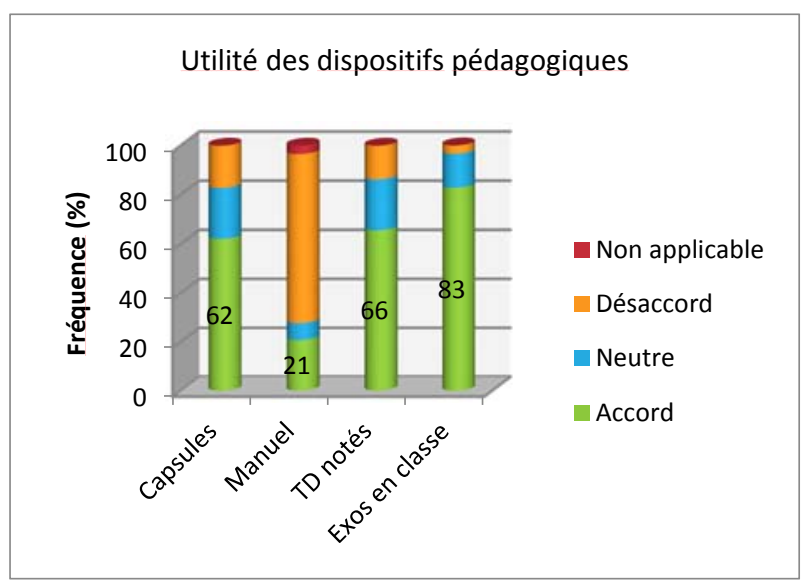

Le cours MEC2420 est un cours de 3 crédits, soit un cours nécessitant 135 heures d'investissement pour l'étudiant, ce qui correspond à la somme des heures de cours, plus les heures de travaux pratiques et les heures de travail personnel. Les étudiants ont indiqué à $64 \%$ que ce nombre d'heures était respecté, 32 \% jugent avoir dépassé les 135 heures et $4 \%$ estiment avoir investi moins d'heures que planifiés. Initialement, nous pensions que les étudiants considèreraient la classe inversée trop exigeante du point de vue du temps requis.

Finalement, est-ce que les étudiants ont apprécié cet enseignement en classe inversée ? La figure 4 résume à l'aide d'un graphique les opinions des étudiants. Ils considèrent que la classe traditionnelle est meilleure ou équivalente à la classe inversée dans une proportion de 67,9\%. Toutefois, 68,9 \% des étudiants perçoivent que la classe inversée permet un apprentissage plus en profondeur ou équivalent que la classe traditionnelle et $75,8 \%$ des étudiants ressentent une plus grande motivation ou une motivation équivalente toujours en comparaison d'une classe traditionnelle. 
Figure 4 Satisfaction de la classe inversée

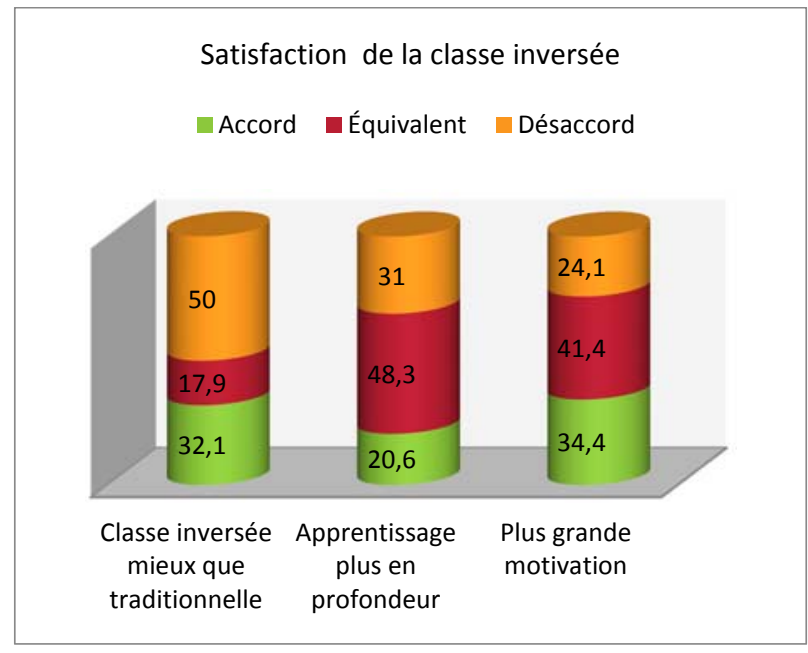

\subsection{CHE0501 - Chimie générale}

En novembre dernier, Polytechnique Montréal a lancé un cours en ligne gratuit et ouvert à tous, (MOOC). Ce cours de 6 semaines, intitulé La chimie, en route vers le génie, permet d'offrir à toute personne possédant quelques bases en mathématiques une introduction à plusieurs concepts de chimie tout en faisant de multiples liens avec l'ingénierie. Ce cours est conçu pour susciter la motivation et l'apprentissage actif. La structure pour les cinq premières semaines est uniforme et combine des capsules vidéos (mot de bienvenue, entrevue avec un chercheur, capsules théoriques, laboratoire) et de nombreux exercices d'apprentissage en lien avec les capsules vidéos. Chacune des semaines s'amorce avec un Mot de bienvenue qui permet de présenter les activités d'apprentissage et les objectifs de la semaine. Par la suite, une Entrevue avec un professeur de Polytechnique Montréal permet de relier les notions qui vont être vues avec une application concrète issue du domaine de l'ingénierie. Puis, des Capsules théoriques viennent expliquer la théorie et présenter divers exemples. Pour faciliter l'écoute de ces capsules, et rendre les étudiants actifs pendant le visionnement, des Fiches synthèses d'aide à la prise de notes sont disponibles. Par la suite, les étudiants peuvent mettre en pratique la théorie grâce à divers Exercices pour lesquels ils obtiennent une rétroaction détaillée immédiate. Un Laboratoire virtuel permet de visualiser une expérience en lien avec le sujet de la semaine, de répondre à des questions et de réaliser des calculs. Finalement, un Retour sur l'entrevue est fait à l'aide de questions et un Test hebdomadaire est proposé. Un examen final récapitulatif vient clore cette formation et une attestation sur l'honneur est délivrée lorsqu'un étudiant obtient une moyenne de $60 \%$ et plus.

Le matériel développé pour ce cours en ligne a permis non seulement de développer un MOOC disponible à toute la communauté, mais aussi de mettre sur pied une classe inversée dans le cadre du cours CHE0501- Chimie générale. Ce cours, offert en année préparatoire, est similaire au cours de chimie générale offert dans le réseau collégial. La classe inversée a été implantée à l'hiver 2016 pour l'un des trois groupes du cours CHE0501, et ce, pendant les 6 premières semaines du trimestre, soit la durée du MOOC développé. Les prochains paragraphes permettent de décrire comment le matériel développé a été utilisé.

\subsubsection{Utilisation du matériel du MOOC en classe inversée}

L'horaire hebdomadaire de la classe traditionnelle du cours de chimie générale est de cinq heures. Trois heures sont consacrées à de l'enseignement magistral et deux heures sont consacrées à la résolution d'exercices en équipe de deux étudiants. À cela s'ajoutent quatre heures de travail personnel.

La classe inversée a également un horaire de cinq heures par semaine, mais dans un contexte misant beaucoup plus sur la participation et l'engagement des étudiants.

Pour chacune des semaines de cours, les étudiants doivent regarder à l'extérieur de la classe les différentes capsules théoriques et préparer leurs questions. Lors de leur arrivée en classe, une partie du temps, environ une heure, est dédiée à répondre à leurs questions. Si peu de questions sont posées, le professeur pose alors des questions de compréhension à tout le groupe. Il est à noter que les premières capsules sont écoutées en classe avec les étudiants pour leur montrer comment les visionner efficacement. Par exemple, nous leur suggérons de rédiger un résumé de la matière et de résoudre les exemples proposés avant d'en écouter la solution. D’ailleurs, nous avons observé que la grande majorité des étudiants ont adopté cette stratégie. Un certain nombre d'entre eux ont consulté le professeur pour faire valider les différents résumés produits.

Par la suite, les étudiants travaillent les exercices proposés, tirés du MOOC, pendant environ deux heures. Ce travail se réalise en équipe de deux étudiants et le professeur circule dans la classe pour les soutenir. Certains exercices sont résolus au tableau et d'autres sont à remettre à la fin du cours. Les deux heures restantes sont consacrées à l'écoute de l'entrevue avec un ingénieur et à 
l'écoute du laboratoire. Ces écoutes sont très interactives et les questions développées dans le cadre du MOOC pour ces deux mécanismes sont utilisées. Ces activités ont créé des discussions animées en classe et ont amené les étudiants à poser une panoplie de questions portant sur les différentes applications reliées au sujet traité et sur les différentes spécialisations en génie. L’implantation de la classe inversée est donc basée sur une grande partie du matériel développé dans le cadre du MOOC. Il a donc été simple de l’implanter.

\subsubsection{Observations}

Cette expérience nous a permis de faire plusieurs constats. Tout d'abord, la très grande majorité des étudiants ont apprécié l’approche de la classe inversée. Certains nous ont mentionné que l'écoute des capsules théoriques à l'extérieur de la classe permet une plus grande flexibilité tant au niveau du moment choisi que de la possibilité de réécouter certains passages plus complexes. De plus, cette approche permet de responsabiliser les étudiants dans leurs études et favorise une participation active en classe. Nous avons d'ailleurs noté un très faible taux d'absentéisme. Ce constat est, selon nous, attribuable aux activités réalisées en classe qui visent toutes à favoriser l'engagement des étudiants.

Nous visons, dans un avenir rapproché, la conception d'un second MOOC relié à la matière abordée lors des 6 autres semaines du cours de chimie générale. Nous serons alors en mesure d'offrir un cours entièrement basé sur l'approche de la classe inversée.

\section{Conclusion}

L'engagement des étudiants dans leur processus d'apprentissage, et à terme une meilleure réussite au cours, est le principal intérêt de l'enseignant pour utiliser des méthodes d'enseignement actives. Les observations relatées dans les deux expériences montrent que la classe inversée peut favoriser cet engagement.

Par ailleurs, le passage d'un mode de classe traditionnel où les étudiants sont passifs à un mode où les étudiants sont actifs nécessite une grande adaptation pour le professeur, mais également pour les étudiants qui montrent une résistance aux changements proposés. Le pourquoi du passage d'une méthode à l'autre, la structure du cours et la contribution de chacune des activités d'enseignement et d'apprentissage doivent être très bien expliqués si l'on veut favoriser l'adhésion à une approche de classe inversée.
Dans la première expérience présentée, on note un effet sur les résultats d'apprentissage chez les étudiants. Toutefois, en dépit de ces résultats favorables, une majorité d’étudiants semble préférer l'approche traditionnelle. Des ajustements au cours, qui tiennent compte, entre autres, des commentaires émis par les étudiants, sont déjà prévus pour la prochaine prestation du cours.

Dans la seconde expérience présentée, au contraire, la majorité des étudiants ont apprécié l'expérience. Le fait que les activités soient tirées d'un MOOC, très structuré, spécifiquement conçu pour favoriser la motivation et l'apprentissage actif, et pour lequel des ressources importantes ont été investies, pourrait être une partie de l'explication. La scénarisation des activités et la mise à l'essai avant l'implantation en classe, dans le cadre d'un cours en ligne, ont peut-être contribué au succès de l'approche auprès des étudiants. 